\title{
A 6-year survey of human brucellosis in a rural area of north-western England and north Wales
}

\author{
Pauline M. Poole \\ M.D., F.R.C.Path., Dip. Bact. \\ Public Health Laboratory, Chester City Hospital, Hoole Lane, Chester
}

\begin{abstract}
Summary
Experiences in the investigation of human brucellosis during a 6-year period are described and the diagnostic value of the various simple laboratory procedures compared. Patients were divided into three groups on the results of the investigations. Patients in groups $I$ and II showed serological evidence varying in degree, which indicated past infection or repeated exposure to infection. Patients in group III were those suffering from active infection during the investigations. The diagnosis, personal information, clinical features and progress of patients in group III are recorded in some detail. Individual case histories of seven veterinary surgeons are given.
\end{abstract}

\section{Introduction}

Registered brucellosis-free herds of cattle increase steadily and the National Eradication Scheme moves slowly toward its goal. Meanwhile pasteurization of milk renders it safe for drinking by the mass of the population. However, a diagnosis of human brucellosis is still frequently under consideration in this laboratory, situated at the centre of a large rural community, in which dairy farming plays a prominent part and where eradication is as yet on a voluntary basis only. Infection is an industrial hazard amongst agricultural workers and veterinary surgeons, whilst a small number of others risk infection from drinking raw milk.

\section{Materials and methods \\ Blood culture}

Five millilitres of freshly drawn whole blood were inoculated into each of two bottles, the first containing diphasic media of Castañeda (Ruiz Castañeda, 1947), the second $50 \mathrm{ml}$ of digest blood with $5 \%$ horse serum, $1 \%$ glucose and bacitracin $10 \mathrm{u} / \mathrm{ml}$, polymixin $4 \mathrm{u} / \mathrm{ml}$ and actidione $0.001 \mathrm{mg} / \mathrm{ml}$. Both cultures were incubated in $10 \% \mathrm{CO}_{2}$ in air at $36-37^{\circ} \mathrm{C}$. Subculture to horse blood agar similarly incubated was carried out after 4,14 and 42 days.

\section{Clot culture}

The clot was separated from blood submitted for serological examination, macerated manually and added to $10 \mathrm{ml}$ of digest broth medium containing additives as for blood culture. Incubation was in $10 \% \mathrm{CO}_{2}$ in air at $36-37^{\circ} \mathrm{C}$, subculture to blood agar being carried out after 14 and 42 days.

\section{Amniotic fluid}

Amniotic fluid was cultured once only, by inoculation of a Castañeda medium and subculture to 'chocolate agar' (Poole, Whitehouse and Gilchrist, 1972).

Successful isolates were of small Gram-negative coccobacilli which conformed with the definition of the genus Brucella and were identified as to species and biotype according to the classification determined by the technical report of the FAO/WHO Expert Committee on Brucellosis (1964). Tests for dye sensitivities were carried out by the method of Cruickshank (1948).

\section{Serology}

The serological tests carried out routinely were (1) the standard agglutination test (SAT) which measures IgG and IgM, (2) the mercaptoethanol test (ME), i.e. agglutination in the presence of 2 mercaptoethanol which destroys IgM but not IgG, (3) the complement fixation test (CFT) detecting IgG and (4) the antihuman globulin (Coombs) test (AHG) for nonagglutinating antibodies either IgG or IgA, all as described by Bradstreet et al. (1970). The Standards Laboratory, Central Public Health Laboratory, London, supplied the suspension of $B$. abortus used in the first three tests and the suspension used in the Coombs' test was obtained from the Central Veterinary Laboratory, Weybridge. For the full interpretation of the tests see Kerr et al. (1968). Considerable difficulty was encountered in obtaining blood for culture so that until clot culture was introduced towards the end of the series, the majority of cases depended for laboratory diagnosis on serological findings. In order to render these of more value, a second specimen of blood for serological examination was requested approximately 4 weeks after initial investigation from all patients considered as query brucellosis. Changes in serological results from no discernible antibody to antibody present, rising 
titres of antibodies, persistence of high titres and the presence of IgM were all observed and their significance assessed in relation to the clinical findings. Sometimes a third or even fourth specimen of serum was valuable at this stage. All serological titres are expressed as reciprocals of serum dilutions.

A titre in the complement fixation test of at least 40 was chosen as an arbitrary requirement where laboratory diagnosis depended wholly on serological tests. IgG alone is involved in the complement fixation test. This test becomes positive more slowly, and commonly does not react at such high titres as are found in the agglutination tests. It falls more quickly and it is not unusual for a positive reaction at high titres to remain for several years in the standard agglutination and antihuman globulin tests owing to the presence of $\operatorname{IgM}$ and $\operatorname{IgA}$, after reactions in the complement fixation test have dropped to 20 or less. The complement fixation test was commenced at a dilution of 20. Once a definite diagnosis of active brucellosis was made, serological reassessment was carried out at intervals as a guide to the activity of the infection, to the success of treatment and in the investigation of possible recurrence of infection.

\section{Results}

The patients

During the 6 years, 299 patients revealed evidence of Brucella infection to some degree. They can be divided into three groups.

Group 1. One hundred and seventy-six patients reacted positively, i.e. with a titre of 20 or higher in the standard agglutination test, but had a titre of less than 20 in the complement fixation test. In those on whom the tests were repeated, no significant change in titre was found. Their symptoms and signs were transient, titres presumably incidental and of an order associated with past infection, possibly subclinical.

Group 2. Sixty-seven patients, as well as reacting positively in the standard agglutination test, also revealed complement fixing titres of at least 20 . Almost certainly there were occasional persons with active infection in this group, but their illnesses were so mild that they were followed up only perfunctorily. It is interesting that amongst this group the titres in the standard agglutination test were higher on average than those in Group I. A high titre in the standard agglutination test generally corresponded with a high titre in the complement fixation test. The majority of these patients were known to be members of the farming community. Of the nine with a titre in the standard agglutination test of $1: 320$ or more, five were farmers, one a veterinary surgeon and one a symptomless child investigated as contact of a suspected case of brucel-
TABLE 1. Titres in standard agglutination and complement fixation tests (all titres being expressed as reciprocals of serum dilutions)

\begin{tabular}{rccccc}
\hline & \multicolumn{3}{c}{ No. of patients } \\
\cline { 2 - 5 } Titres & \multicolumn{2}{c}{ Group I } & & Group II \\
\cline { 2 - 5 } \cline { 3 - 5 } & SAT & CFT & & SAT & CFT \\
\hline 20 & 64 & - & 5 & 23 \\
40 & 64 & - & 14 & 26 \\
80 & 40 & - & 25 & 12 \\
160 & 4 & - & 14 & 5 \\
320 & 4 & - & 3 & 1 \\
640 & - & - & 3 & - \\
1280 & - & - & 3 & - \\
\hline
\end{tabular}

losis in a farming family. The occupations of the remaining two were unknown.

Table 1 shows the distribution of patients by serological findings in groups I and II.

Group 3. Fifty-six patients were identified as suffering from active brucellosis, either acute or chronic. The diagnosis was made on one or more criteria: isolation of $B$. abortus, rising titres, presence of IgM as well as IgG or continuous raised titres (Table 2).

\section{Patients with active brucellosis in group III}

Distribution of cases by time. The cases occurred throughout the 6 years. The total number in any one year was not high, six in 1967 and 1968, rising to nine in 1969 and 1970, with a peak of twelve in 1971. The numbers fell to eight in 1972 and six in 1973.* Four more patients were originally infected at varying times before 1967, but were re-investigated during the years in question. The apparent increase in infection in 1968, 1970 and 1971 was coincident with the aftermath of the outbreak of foot and mouth disease in the winter of 1968-69 and the consequent movement of stock as herds were replenished.

Sex and age. Forty-five $(80 \%)$ patients were male and eleven $(20 \%)$ female. Infection occurred only twice in children under 15 years and in patients of more than 54 years. Thirty-three $(59 \%)$ were young active adults between 15 and 34 years, and nineteen $(34 \%)$ between 35 and 54 years.

Occupation. Thirty-five $(62 \%)$ patients were either farmers, farm workers or members of farming families. Seven $(12 \%)$ were veterinary surgeons and the work of two others took them frequently to farms. Nine had no occupational hazard. No information was available on the remaining three.

Raw milk, contact with cows. Twenty-nine (52\%) patients were known to drink raw milk and on fourteen occasions $B$. abortus was isolated from the corresponding milk. Five persons denied drinking raw milk. No definite history was available

* Two only of these six cases included here. 
TABLE 2. Summary of patients in group III

\begin{tabular}{|c|c|c|c|c|c|c|c|c|c|}
\hline \multirow[b]{2}{*}{ Case no. } & \multirow[b]{2}{*}{ Sex } & \multirow[b]{2}{*}{ Age } & \multirow[b]{2}{*}{$\begin{array}{l}\text { Occupational } \\
\text { hazard }\end{array}$} & \multirow[b]{2}{*}{$\begin{array}{l}\text { Raw } \\
\text { milk }\end{array}$} & \multirow[b]{2}{*}{ Culture } & \multicolumn{4}{|c|}{ Diagnostic criterion } \\
\hline & & & & & & $\begin{array}{c}\text { Rising } \\
\text { titre }\end{array}$ & $\begin{array}{l}\text { Presence } \\
\text { of IgM }\end{array}$ & $\begin{array}{c}\text { High } \\
\text { titre }\end{array}$ & Other \\
\hline 1 & $\mathbf{M}$ & 19 & + & $?$ & + & + & + & & \\
\hline 2 & M & 23 & + & + & + & & + & & \\
\hline 3 & $\mathrm{~F}$ & 24 & + & + & + & & + & & \\
\hline 4 & $\mathbf{M}$ & 54 & + & + & + & & + & & \\
\hline 5 & $\mathbf{M}$ & 23 & + & + & + & & & + & \\
\hline 6 & M & 31 & + & - & + & & & + & \\
\hline 7 & M & 55 & - & ? & + & & + & & \\
\hline 8 & $\mathbf{M}$ & 40 & $?$ & + & + & & + & & \\
\hline 9 & $\mathbf{M}$ & 32 & - & + & + & & + & & \\
\hline 10 & $\mathbf{M}$ & 25 & - & + & + & + & + & & \\
\hline 11 & $\mathbf{M}$ & 32 & + & $?$ & $\stackrel{+}{+}$ & & & $\begin{array}{c}+ \\
(1967)\end{array}$ & \\
\hline 12 & $\mathbf{M}$ & 40 & + & $?$ & + & & & & \\
\hline 13 & $\mathbf{M}$ & 23 & + & + & & + & & & \\
\hline 14 & $\mathbf{M}$ & 29 & + & + & & + & & & \\
\hline 15 & $\mathbf{M}$ & 51 & + & - & & + & & & \\
\hline 16 & $\mathbf{F}$ & 22 & - & + & & + & & & \\
\hline 17 & F & 19 & + & - & & + & & & \\
\hline 18 & $\mathbf{F}$ & 23 & + & + & & + & & & \\
\hline 19 & $\mathbf{M}$ & 26 & + & $?$ & & + & & & \\
\hline 20 & M & 24 & + & $?$ & & + & & & \\
\hline 21 & $\mathbf{M}$ & 24 & + & $?$ & & + & & & \\
\hline 22 & $\mathbf{M}$ & 32 & + & ? & & & & + & \\
\hline 23 & F & 31 & + & $?$ & & & & + & \\
\hline 24 & F & 47 & + & + & & & & + & \\
\hline 25 & $\mathbf{M}$ & 47 & + & $?$ & & & & & + \\
\hline 26 & $\mathbf{M}$ & 46 & - & + & & & + & & $T$ \\
\hline 27 & $\mathbf{F}$ & 44 & $?$ & + & & & + & & \\
\hline 28 & $\mathbf{F}$ & 48 & + & + & & & $r$ & + & \\
\hline 29 & $\mathbf{M}$ & 30 & - & + & & & + & $T$ & \\
\hline 30 & $\mathbf{F}$ & 17 & + & + & & & & + & \\
\hline 31 & $\mathbf{F}$ & 39 & - & + & - & & + & & \\
\hline 32 & $\mathbf{M}$ & 22 & + & $?$ & - & & + & & \\
\hline 33 & $\mathbf{M}$ & 32 & + & + & - & & + & & \\
\hline 34 & M & 33 & + & - & & & + & & \\
\hline 35 & $\mathbf{M}$ & 53 & $?$ & $?$ & - & & & + & \\
\hline 36 & $\mathbf{M}$ & 31 & + & + & - & & & + & \\
\hline 37 & $\mathbf{M}$ & 40 & + & + & - & & + & & \\
\hline 38 & $\mathbf{M}$ & 35 & + & ? & - & & + & & \\
\hline 39 & $\mathbf{M}$ & 21 & + & + & - & & & + & \\
\hline 40 & $\mathbf{M}$ & 60 & + & $?$ & - & & & + & \\
\hline 41 & $\mathbf{M}$ & 24 & + & ? & - & & & + & \\
\hline 42 & $\mathbf{M}$ & 42 & + & ? & - & + & + & & \\
\hline 43 & $\mathbf{M}$ & 21 & + & + & - & & & + & \\
\hline 44 & $\mathbf{M}$ & 18 & + & + & - & & + & & \\
\hline 45 & $\mathbf{M}$ & 21 & + & + & - & & & + & \\
\hline 46 & $\mathbf{M}$ & 19 & + & ? & - & & & + & \\
\hline 47 & $\mathbf{F}$ & 10 & + & + & - & & + & r & \\
\hline 48 & $\mathbf{M}$ & 35 & - & $?$ & - & & $T$ & + & \\
\hline 49 & $\mathbf{M}$ & 22 & + & $?$ & - & & + & & \\
\hline 50 & $\mathbf{M}$ & 41 & + & $?$ & - & & & + & \\
\hline 51 & $\mathbf{M}$ & 28 & + & ? & - & & & $T$ & + \\
\hline 52 & $\mathbf{M}$ & 7 & - & + & - & & & + & \\
\hline 53 & $\mathbf{M}$ & 51 & + & $?$ & - & & & + & \\
\hline 54 & $\mathbf{M}$ & 52 & + & + & - & & + & & \\
\hline 55 & $\mathbf{M}$ & 26 & + & + & - & + & & & \\
\hline 56 & $\mathbf{M}$ & 51 & + & - & - & + & & & \\
\hline
\end{tabular}


from twenty-two. Forty-two $(75 \%)$ patients had contact with cows, twenty-three being associated with proved infected herds. It was not always possible to obtain this information. Unless milk was being sold raw to the public, investigation was at the farmer's discretion and samples were not necessarily submitted for testing. Nine patients had no contact with cows. No information was available from five. In all, fifty-one patients $(91 \%)$ either drank raw milk and/or had contact with cows.

Clinical features. The degree of illness varied from very mild, constituting a short pyrexial episode only, to severe enough to require hospitalization. Four patients suffered chronic ill health. Although full clinical information was not received from every patient, it was possible from the details available to recognize the clinical profile of the disease. Malaise, pyrexia and sweating predominated. Sore throat, headache, arthralgia, myalgia, shivering or influenza-like illness were common complaints. Likewise loss of appetite, loss of weight and depression were frequently described. Pyrexia was usually intermittent, and all symptoms were repeatedly described as being more troublesome at night. The signs observed included splenomegaly on nine occasions and adenopathy on five. Rashes were recorded twice. The symptoms and signs were those typically associated with brucellosis. Rather more unusual were one patient who aborted (Poole et al., 1972), one with orchitis, two with iridocyclitis and one suffering from diplopia and slurred speech.

Onset history. The aetiology of the illness was suspected within 1 week of onset in four patients, between 1 and 4 weeks in eleven, and between 5 and 12 weeks in nine. The onset history was described as short in a further fifteen. Fourteen patients had, however, been ill for more than 12 weeks, four of these for about 2 years and three for many years. No information about onset was available from three patients.

\section{Diagnostic criteria and clinical progress}

Culture was carried out in twenty-one cases and $B$. abortus isolated from twelve, an isolation rate of $57 \%$. Five strains were identified as biotype 1 , two as biotype 2 . Eight of fourteen blood cultures were successful, four in the Castañeda culture and three in the digest broth. The medium was not recorded in the eighth isolation. The most rapid isolation was after 4 days' incubation before subculture and the slowest, 6 weeks. Six clot cultures were prepared, three proving successful. The twelfth isolation was from amniotic fluid. Eleven isolates were from patients with a clinical history of illness for between 2 days and 6 months. A twelfth was from a veterinary surgeon who suffered a chronic low grade illness for many years before investigation.
Nine patients responded satisfactorily to treatment, one developed evidence of chronicity with high serological titres, one suffered a relapse after 5 months and one was still unwell when last observed.

Rising titre. Twelve patients were diagnosed by demonstration of a rise in titre, four-fold or more, in the standard agglutination test, and/or the complement fixation test in two specimens taken at an interval of approximately 1 month, always provided that the complement fixation test was at least 40 or more on at least one occasion.* Clinical results were good throughout this group. Titres subsided satisfactorily in all but three, two of whom were veterinary surgeons and one a farmer still under observation. Rising titres were also demonstrated in two patients from whom $B$. abortus was isolated.

Presence of IgM. Thirteen patients were diagnosed by the demonstration of the presence of specific IgM as well as IgG, both being present simultaneously in active infection (Coghlan and Weir, 1967). A decrease in titre at least fourfold between the standard agglutination test and the mercaptoethanol test, with the usual proviso of the complement fixation test being positive at a titre of 40 or more, was the arbitrary standard chosen to select these patients. In ten the onset was short, and in three prolonged. All eventually settled to low titres.

Example. A farmer aged 33 years with a known infected herd gave a short history of onset of an influenzal illness followed by depression. Titres at this stage were SAT 1280, ME 320, CFT 640, AHG 1280. He was well after a 4-week course of tetracycline, although there was little change in serology, SAT 640, ME 320, CFT 640, AHG 1280. Five months later the titres had fallen, SAT 160, ME 160, CFT 20, AHG 160 and he remained well.

As well as the thirteen patients diagnosed on this criterion, eight of the twelve patients from whom $B$. abortus was isolated and in whom, therefore, the infection was proved beyond doubt, showed this phenomenon.

High titres. Raised titres in all tests have been associated with chronic brucellosis or asymptomatic infection (Coghlan and Weir, 1967). Specific IgM is absent from the blood of such patients. As a result of constant re-exposure of persons in the dairy community, the presence of high titres in all tests can be demonstrated in normal health (Kerr et al., 1968; Henderson and Hill, 1972). All other possible causes for symptoms and signs must therefore be eliminated before concluding that a patient is indeed suffering from active brucellosis. Titres of this kind were the only initial evidence in seventeen patients. Ten cases were of recent onset. Seven had a long history of either vague ill health or repeated attacks of 'influenza'. Two of these seven had indeed previous

* Arbitrary titre. 
serological evidence of infection several years earlier, and probably represented chronic infection. The five remaining were more likely to have been the result of delayed investigation. Sixteen patients in this diagnostic category made a good clinical recovery. However, in two, both veterinary surgeons, the serological titres remained high. The seventeenth patient continued to complain three years later, although serologically satisfactory. As well as these seventeen, two patients from whom $B$. abortus was isolated had high titres in all tests when first investigated.

Two patients did not fill any of the foregoing requirements. However, both had suspicious symptoms and although their titres were not high at any time, a fourfold fall in the complement fixation test coinciding with return to health was observed on serial examination. On these grounds they were all included as cases of active infection. One was a veterinary surgeon and will be described in detail later.

\section{Treatment progress}

Treatment. Tetracycline, streptomycin and co-trimoxazole were the drugs commonly used. However, the wide variations in both dosage and duration of treatment prevent any assessment of their relative efficiency, when used either alone or in combination. Twenty-six patients were given tetracycline only, fifteen were treated with tetracycline in combination with streptomycin and seven received co-trimoxazole, either alone or in addition to tetracycline and/or streptomycin. Six patients were treated for less than 4 weeks, twenty-one for more than 4 weeks but less than twelve. Sixteen were treated for longer than twelve weeks. Details about the duration of treatment are not known for five patients; four others received no specific treatment and there is no information on the treatment of the remaining four.

Clinical recovery. Tables 3 and 4 illustrate the interval between initial investigation and return to subjective feeling of well-being in relation to type and duration of treatment. Thirty-five $(62 \%)$ of the forty-nine patients on whom this information is available were well within 3 months; this in an infection renowned for chronicity. Only three were positively known to have symptoms persisting for more than 1 year. Of the thirteen patients well within 4 weeks, twelve had an onset of short duration. $B$. abortus was isolated from four of them and two were diagnosed by rising titres. The thirteenth patient was ill for 18 months before investigation. Four of these patients received tetracycline alone, five a combination of tetracycline and streptomycin, one co-trimoxazole and tetracycline and one co-trimoxazole alone. One patient was untreated and no information is available about the thirteenth. Two patients were treated for less than 4 weeks, seven for between 4 weeks and 3 months and two for more than 3 months.

Twenty-two patients regained normal health within between 4 weeks and 3 months; seventeen of these

TABLE 3. Time from commencement of investigation to return to subjective well-being in relation to therapeutic agent used

\begin{tabular}{lccccc}
\hline \multirow{2}{*}{ Treatment } & \multicolumn{5}{c}{ Return to health } \\
\cline { 2 - 6 } & $\begin{array}{c}\text { 4 weeks } \\
\text { or less }\end{array}$ & $\begin{array}{c}\text { 4 weeks- } \\
\text { 3 months }\end{array}$ & $\begin{array}{c}\text { 3 months- } \\
\text { 1 year }\end{array}$ & > 1 year & Not known \\
\hline Tetracycline & 4 & 7 & 9 & 2 & 4 \\
Tetracycline+streptomycin & 5 & 9 & 0 & 1 & 0 \\
Co-trimoxazole+/-other antibiotics & 2 & 3 & 1 & 0 & 1 \\
No treatment & 1 & 2 & 1 & 0 & 0 \\
Treatment unknown & 1 & 1 & 0 & 0 & 2 \\
Total & 13 & 22 & 11 & 3 & 7 \\
\hline
\end{tabular}

TABLE 4. Time from commencement of investigation to return to subjective well-being in relation to duration of treatment

\begin{tabular}{lccccc}
\hline & \multicolumn{5}{c}{ Return to health } \\
\cline { 2 - 6 } $\begin{array}{l}\text { Duration of } \\
\text { treatment }\end{array}$ & $\begin{array}{c}\text { 4 weeks } \\
\text { or less }\end{array}$ & $\begin{array}{c}\text { 4 weeks- } \\
\text { 3 months }\end{array}$ & $\begin{array}{c}\text { 3 months- } \\
\text { 1 year }\end{array}$ & >1 year & Not known \\
\hline <4 weeks & 2 & 2 & 1 & 1 & 0 \\
4 weeks to 3 months & 7 & 9 & 3 & 1 & 1 \\
$>3$ months & 2 & 6 & 4 & 1 & 3 \\
Duration not known & 0 & 2 & 2 & 0 & 1 \\
No treatment & 1 & 2 & 1 & 0 & 0 \\
Treatment unknown & 1 & 1 & 0 & 0 & 2 \\
Total & 13 & 22 & 11 & 3 & 7 \\
\hline
\end{tabular}


had an illness of short onset and five, of whom two were veterinary surgeons, a prolonged onset. $B$. abortus was isolated from six patients, and three showed rising titres. In this group seven patients were given tetracycline, nine tetracycline with streptomycin, two co-trimoxazole with streptomycin and tetracycline, and one co-trimoxazole alone. Two patients were untreated and there are no details about the remaining patient. Two were treated for less than 4 weeks, nine for between 4 weeks and 3 months and six for more than 3 months. The duration of treatment is unknown in the remaining two.

Eleven patients took between 3 and 12 months to regain health. Nine of these had an illness of short onset, two showed rising titres. The tenth, a woman, was ill for 2 years before diagnosis. The eleventh was of special interest. He was a dairy farmer aged 41, who had inoculated himself with S19 accidentally in 1955 , at which time he was reported to have a titre of 160 in the standard agglutination test. He developed iridocyclitis and, when he came to notice again 12 years later during this investigation, complained of recurrent malaise, and joint pains. His spleen was palpable at this stage. Serological investigation revealed SAT 320, ME 160, CFT 80, AHG 320. He was treated with tetracycline daily for 2 weeks and then intermittently for 8 months. When last tested in 1973 he had SAT 160, CFT 20, AHG 320. Other than the residual effects of iridocyclitis he was well. Eight further patients received tetracycline alone and one co-trimoxazole. One was untreated. One patient was treated for less than 4 weeks, three for between 4 weeks and 3 months. As well as the man discussed at length, three more patients were treated for longer than 3 months. The duration of treatment is unknown for the two remaining.

Three patients continued to have symptoms for longer than 12 months. Two, one of whom was a veterinary surgeon, recovered in 15 months, one being treated with tetracycline for 4 weeks, the other for more than 3 months. The third man was still not well after 3 years, although he had a course of treatment with tetracycline and streptomycin lasting 8 weeks.

The choice of treatment largely depended on the preferences of the clinician concerned. To some extent continued symptoms resulted in prolonged treatment. It is, however, of interest that four patients recovered without treatment, one within 4 weeks, two more within 3 months and one within a year. All suffered from mild illness of short onset. Three were farmers, one a veterinary surgeon.

Fall in titre. Once the diagnosis was made and treatment initiated, serial examination of the patient's serum was carried out to determine the response of the infection to treatment. If all was well,
TABLE 5. CF titres at final examination in relation to time from commencement of investigation of illness

\begin{tabular}{rccc}
\hline CFT & $<1$ year & $1-2$ years & $>2$ years \\
\hline$<20$ & 10 & 5 & 7 \\
20 & 6 & 5 & 4 \\
40 & 3 & 4 & 1 \\
80 & 1 & 2 & 2 \\
160 & 1 & - & - \\
320 & 1 & 2 & - \\
640 & 1 & - & - \\
1280 & - & - & 1 \\
2560 & - & & \\
\hline
\end{tabular}

titres gradually fell in all tests. However, the complement fixation test reflecting the presence of IgG only, fell to lower levels sooner than the other tests, and so provided a useful indication on the activity of the infection (Table 5). A complement fixation test of $<20$ was the aim, although not all patients were monitored for a sufficiently long time to achieve this low level. Twenty-two patients in fact reached this titre during the investigation, ten within 1 year, five more within 2 years and the remainder in more than 2 years. Fifteen more patients had a complement fixation test of 20 on the final observation, six within 1 year, five within 2 years and four in longer than 2 years. A further eight patients had a complement fixation test of $\mathbf{4 0}$ on the last check. These were probably falling satisfactorily, although not followed up further. One patient took more than 2 years to fall to this titre. The eleven remaining patients had final titres between 80 and 2560, three of them having been followed for more than 2 years. Five of these eleven were veterinary surgeons and exposed to repeated infective stimuli which may well result in prolonged high titres.

Recurrence of infection. Four patients were probably suffering from chronic infection when first observed in this investigation. Three were veterinary surgeons and one the farmer with iridocyclitis described earlier. Amongst the remaining patients in whom the diagnosis was suspected for the first time, eleven who were apparently settling down satisfactorily had further symptoms, which were consistent with renewed activity of the infection. However, on rechecking the titres, ten of these showed no evidence of reactivation and the symptoms did not persist.

The eleventh suffered a genuine recurrence. A twenty-five-year-old bricklayer who drank raw milk, had a history of 2 weeks' pyrexia when first seen. $B$. abortus type 1 was isolated from blood cultures and serology at this time was SAT 5120, ME 160, CFT 320, AHG 10,240. He was treated with tetracycline for 8 weeks, feeling well again within 4 weeks, his titres falling to SAT 640 , ME 160, CFT 320, AHG 1280. He remained well for 3 months, 
before developing a further pyrexial illness with a concurrent rise in titres SAT 2560, ME 1280, CFT 2560, AHG 2560. Blood cultures at this stage yielded no growth. He soon felt well again, further treatment being given with streptomycin for 2 weeks and tetracycline for 7 weeks. Two years from the onset of the original illness, the titres were SAT 160, CFT 20, AHG 160.

\section{Veterinary surgeons}

Seven patients were veterinary surgeons. Three had previous history of infection before the years under investigation. The first of these, 32 years old at onset, yielded the organism from blood cultures during an original acute illness in 1963. At that time the standard agglutination test was $<20$. He was treated with streptomycin for 1 week and tetracycline for 3 weeks. During the following 4 years he suffered from niggling ill health and was given tetracycline intermittently with some relief. Serological investigations were not carried out again until 1967, when he had a further pyrexial illness. Titres then were SAT 1280, ME 1280, CFT 320, AHG 5120. No precise details of treatment are available, but 1 year later the titres had risen to SAT 5120, CFT 2560, AHG 10,240, coincident with an attack of iridocyclitis. One month later all titres had fallen by one dilution. Since that time he is stated to have remained fit, although no further laboratory investigation has been carried out.

The second, a man aged 40 years, had a vague history of brucellosis for several years unsupported by laboratory evidence. At intervals he complained of malaise, which usually improved after a holiday. During one such incident in 1970, blood was submitted for serology and culture. The serological findings SAT $<20$, CFT $<20$, AHG 20, did not suggest infection, but $B$. abortus was isolated from blood culture. One month later the serology was unchanged. He was treated with tetracycline for 3 months and was stated to be fit on enquiry 2 years later.

The third, a man of 60 years, presented with a story of several months' intermittent fever, malaise, sweating and loss of weight. He suffered transient rashes after delivering calves. An SAT carried out 6 years before during a routine survey, was positive at a titre of 30. When investigated at the time of his illness titres were SAT 320, ME 320, CFT 320, AHG 640. His circumstances were such that he continued working and despite 2 months' treatment with tetracycline was not improving. The symptoms were undulant and worse at night. He was then given streptomycin $1 \mathrm{~g}$ daily for 2 weeks, with tetracycline for 3 weeks. After an interval of 1 week without treatment he had a further course of tetracycline for 4 weeks. He gradually began to feel better. Eight months later his titres were SAT 160 , ME 80, CFT 160, AHG 640. He retired and was well at that time.

Two more veterinary surgeons were diagnosed by rising titres, their illnesses being of recent onset. One treated with streptomycin for 2 weeks, and tetracycline for 2 months was soon well and remained so after 7 months, although his titres continued to be high, SAT 1280, CFT 640, AHG 2560. The other, treated originally with tetracycline only for 4 weeks, was still unwell 10 months later, SAT 1280, CFT 320, AHG 5120. A prolonged intermittent course of tetracycline was given and 16 months from onset he left the district apparently well. Titres remained quite high, SAT 320, ME 160, CFT 320, AHG 640.

The illness in the sixth veterinary surgeon, a man of 51 years who complained of intermittent pyrexia, aching, and depression, of 6 months' duration, was originally considered to be largely functional, although the titres were SAT 160, ME 160, CFT 320, AHG 640. Blood culture was negative. However, his spleen became palpable and he was given a short course of streptomycin with tetracycline, followed by intermittent tetracycline on 2 days weekly. He felt fit within 2 months, although his titres remained unchanged and he was still taking tetracycline 12 months later.

The seventh was an American veterinary surgeon working at a turkey farm in this country. He consulted a doctor, complaining of sore throat and malaise of 4 weeks' duration. He was adamant that he had never been in contact with infected cattle. He had, however, used S19. Titres at this time were SAT 160, ME 20, CFT 20, AHG 320. IgM was therefore present, although the CFT did not reach the titre required under the criteria described. $\mathrm{He}$ settled down without treatment and 5 months later his CFT had fallen to 5, although there was little change in other titres, SAT 80, ME 20, AHG 160.

All the veterinary surgeons were male, in five the titres were still raised when apparently restored to health. Although B. abortus was isolated from the second veterinary surgeon described, his illness was chronic in character. All proved difficult to follow up, apparently suffering from an occupational reluctance to investigation.

\section{Discussion}

The methods used here for the investigation of suspected brucellosis were simple, and for the most part routine. However, on the results achieved it was generally possible to make a diagnosis with some confidence. Isolation of $B$. abortus was of course conclusive. Where this was attempted there was a success rate of $57 \%$. Unfortunately, blood cultures were received from only $25 \%$ of patients. There is considerable reluctance to take blood 
cultures, the popular reputation of the organism being that it is difficult to isolate. This discourages doctors from making the effort but, as these results show, it is well worthwhile. Clot culture may to some extent compensate for the omission of blood cultures, although the numbers examined so far are small. It is certainly of some value, is easily carried out and can result in an unequivocal diagnosis. Next in value is the demonstration of rising serological titres. Sometimes, however, it is necessary to carry out several such examinations before a rise is revealed, or infection excluded. The ME test has been found to be valuable in this laboratory when considered in relation to the other serological tests. The demonstration of the presence of specific IgM indicates active infection provided the presence of IgG can also be displayed. It was present in eight of twelve patients from whom $B$. abortus was isolated, a finding which gives support to its value in diagnosis. It can, however, be found without coincident IgG in the serum of some patients for several years after the infection is overcome.

High titres in all tests are more difficult to interpret. They are sometimes found in healthy members of the rural community. However, provided all other possible aetiology is carefully excluded and if both symptoms and titres persist, it is reasonable to treat the patients on such results. A return to health accompanied by a fall in titres vindicates this method of diagnosis. High titres were found in two patients from whom $B$. abortus was isolated, one of these patients having been ill for only 2 weeks. The absence or presence of antibody in low titres only, does not exclude infection. One of the veterinary surgeons had minimal titres and value symptoms only, and might well have been overlooked. The isolation of $B$. abortus was conclusive in this case. No doubt other such cases occur. In two further cases described it was not possible to adhere rigidly to the defined criteria for a diagnosis. Each had to be assessed individually. In one, by carrying out complement fixation tests at dilutions commencing at 5 instead of 20 , it was possible to show a fall in titre. The routine test has now been amended to commence the complement fixation test at this lower dilution, and is useful in detecting the presence of the antibody early in the disease, so that there is no delay in treatment. The awareness of clinicians of the possible aetiological role of $B$. abortus resulted in a diagnosis in several instances when the symptoms in themselves might well have been disregarded, the patients' complaints being almost trivial. This investigation shows clearly that brucellosis may present in the laboratory as an infection of all grades of severity, patients falling into three groups on the result of the several tests. Patients in Group I with low level of antibody were not currently ill but had suffered from infection in the past; those in Group II had rather more antibody, possibly owing to more recent infection or as a result of possible subclinical contact with the antigen in their daily environment. Patients in Group III were suffering from active infection at the time of investigation and required treatment.

It is important that once diagnosed, the patient's progress be monitored serologically as well as clinically. This enables the success or otherwise of individual treatment to be evaluated and adjusted as necessary. Any tendency to chronicity can be tackled. At the same time it allows a rational assessment of any further suspicious symptoms. The complement fixation test revealing the presence of IgG is a valuable index of change in activity of infection. Complement fixation titres generally fall to a low level more quickly than titres in other tests, which may linger for many years. Veterinary surgeons are the exception. Their titres tend to remain high indefinitely, despite apparent clinical recovery. This is the result of repeated re-exposure to antigenic stimulus from infected tissue. Members of this group often complain of fleeting rashes with a pyrexial episode after such exposure, a reaction of hypersensitivity.

\section{References}

Bradstreet, C.M.P., Tannahill, A.J., Pollock, T.M. \& MOGFORD, H.E. (1970) Intradermal and serological tests in suspected Brucella infection in man. Lancet, ii, 653.

Coghlan, J.D. \& Weir, D.M. (1967) Antibodies in human brucellosis. British Medical Journal, ii, 269.

Cruickshank, J.C. (1948) A simple method for testing dye sensitivity of Brucella species. Journal of Pathology and Bacteriology, 60, 328.

Henderson, R.J. \& Hill, D.M. (1972) Clinical ProblemsSubclinical Brucella Infection in Man. British Medical Journal, iii, 154.

JoINT FAO/WHO EXPERT COMMITTEE ON BRUCELlosis (1964) Fourth Report Technical Report Series. World Health Organization, No. 289.

KerR, W.R., McCaughey, W.J., Coughlan, Joyce D., Payne, D.J.H., Quaife, R.A., Robertson, L. \& FARrell, I.D. (1968) Techniques and interpretations in the serological diagnosis of brucellosis in man. Journal of Medical Microbiology, 1, 181.

Poole, P.M., Whitehouse, D.B. \& Gilchrist, M.M. (1972) A case of abortion consequent on infection with Brucella abortus biotype 2. Journal of Clinical Pathology, 25, 882.

Ruiz Castaneda, M. (1947) A Practical method for routine blood cultures in brucellosis. Proceedings of the Society for Experimental Biology (N.Y.), 64, 114. 